\title{
Impaired neuromuscular transmission and skeletal muscle fiber necrosis in mice lacking $\mathrm{Na} / \mathrm{Ca}$ exchanger 3
}

\author{
Sophie Sokolow, ${ }^{1,2}$ Mario Manto, ${ }^{3}$ Philippe Gailly, ${ }^{4}$ Jordi Molgó, ${ }^{5}$ Clarisse Vandebrouck, ${ }^{4}$ \\ Jean-Marie Vanderwinden, ${ }^{6}$ Andre Herchuelz, ${ }^{2}$ and Stéphane Schurmans ${ }^{1}$ \\ ${ }^{1}$ Institut de Recherches en Biologie Humaine et Moléculaire-Institut de Biologie et de Médecine Moléculaires \\ (IRIBHM-IBMM), Université Libre de Bruxelles, Gosselies, Belgium \\ ${ }^{2}$ Laboratory of Pharmacology and Therapeutics and \\ ${ }^{3}$ Laboratory of Experimental Neurology, Université Libre de Bruxelles, Brussels, Belgium \\ ${ }^{4}$ Department of Physiology, Université Catholique de Louvain, Brussels, Belgium \\ ${ }^{5}$ Institut Fédératif de Neurobiologie Alfred Fessard, Laboratoire de Neurobiologie Cellulaire et Moléculaire, \\ Unité Propre de Recherche 9040, Centre National de la Recherche Scientifique, Gif-sur-Yvette, France \\ ${ }^{6}$ Laboratory of Neurophysiology, Université Libre de Bruxelles, Brussels, Belgium
}

\begin{abstract}
We produced and analyzed mice deficient for $\mathrm{Na} / \mathrm{Ca}$ exchanger 3 (NCX3), a protein that mediates cellular $\mathrm{Ca}^{2+}$ efflux (forward mode) or $\mathrm{Ca}^{2+}$ influx (reverse mode) and thus controls intracellular $\mathrm{Ca}^{2+}$ concentration. NCX3-deficient mice $\left(\mathrm{Ncx3}^{-/-}\right)$present a skeletal muscle fiber necrosis and a defective neuromuscular transmission, reflecting the absence of NCX3 in the sarcolemma of the muscle fibers and at the neuromuscular junction. The defective neuromuscular transmission is characterized by the presence of electromyographic abnormalities, including low compound muscle action potential amplitude, a decremental response at low-frequency nerve stimulation, an incremental response, and a prominent postexercise facilitation at high-frequency nerve stimulation, as well as neuromuscular blocks. The analysis of quantal transmitter release in $\mathrm{Ncx}^{-/-}$neuromuscular junctions revealed an important facilitation superimposed on the depression of synaptic responses and an elevated delayed release during high-frequency nerve stimulation. It is suggested that $\mathrm{Ca}^{2+}$ entering nerve terminals is cleared relatively slowly in the absence of $\mathrm{NCX} 3$, thereby enhancing residual $\mathrm{Ca}^{2+}$ and evoked and delayed quantal transmitter release during repetitive nerve stimulation. Our findings indicate that NCX3 plays an important role in vivo in the control of $\mathrm{Ca}^{2+}$ concentrations in the skeletal muscle fibers and at the neuromuscular junction.
\end{abstract}

J. Clin. Invest. 113:265-273 (2004). doi:10.1172/JCI200418688.

\section{Introduction}

In order to avoid prolonged elevation of cytosolic free calcium concentration $\left(\left[\mathrm{Ca}^{2+}\right]_{i}\right)$, cells use multiple channels, pumps, exchangers, and chelators (1). The Na/Ca exchanger $(\mathrm{NCX})$ is a plasma membrane protein responsible for $\mathrm{Ca}^{2+}$ extrusion from cells. Indeed, in its forward mode, NCX extrudes one $\mathrm{Ca}^{2+}$ against three

Received for publication April 17, 2003, and accepted in revised form November 19, 2003.

Address correspondence to: Sophie Sokolow, IRIBHM-IBMM, Université Libre de Bruxelles, rue des Professeurs Jeener et Brachet 12, B-6041 Gosselies, Belgium. Phone: 32-2-650-98-24; Fax: 32-2-650-98-20; E-mail: ssokolow@ulb.ac.be.

Conflict of interest: The authors have declared that no conflict of interest exists.

Nonstandard abbreviations used: electromyography (EMG); cytosolic free calcium concentration $\left(\left[\mathrm{Ca}^{2+}\right]_{\mathrm{i}}\right) ; \mathrm{Na} / \mathrm{Ca}$ exchanger (NCX); neuromuscular junction (NMJ); vesicular acetylcholine transporter (VAChT); plasma membrane $\mathrm{Ca}^{2+}$ ATPase type 1 (PMCA1); flexor digitorum brevis (FDB); $N$-methyl-D-glucamine (NMDG); Evans blue dye (EBD); compound muscle action potential (CMAP); hypoxanthine phosphoribosyltransferase (HPRT); single-fiber electromyography (SFEMG); single-fiber action potential (SFAP); mean consecutive discharge (MCD); evoked endplate potential (EPP); spontaneous miniature endplate potential (MEPP); embryonic stem (ES); NCX3 immunoreactivity (NCX3-ir); sarcoplasmic reticulum (SR); 2,5di(tert-butyl)-1,4-benzohydroquinone (TBQ); $\mathrm{SR} \mathrm{Ca}^{2+}$ ATPase (SERCA); repetitive nerve stimulation (RNS); low rate of repetitive stimulation (LRRS); high rate of repetitive stimulation (HRRS). entering $\mathrm{Na}^{+}$, using the $\mathrm{Na}^{+}$gradient across the plasma membrane as the energy source $(2,3)$. In view of its high exchange capacity, NCX is well suited for rapid recovery from high $\left[\mathrm{Ca}^{2+}\right]_{i}$ and may play an important role in maintaining $\mathrm{Ca}^{2+}$ homeostasis and protecting cells from $\mathrm{Ca}^{2+}$ overload and death $(2,4)$. In its reverse mode, NCX allows $\mathrm{Ca}^{2+}$ entry in cells (2).

Three isoforms of the exchanger (NCX1, NCX2, and NCX3) have been cloned and are encoded by distinct genes (4-6). NCX1 is most abundant in heart but is widely distributed in most cells $(5,7)$. NCX1 is required for $\mathrm{Na}^{+}$-dependent $\mathrm{Ca}^{2+}$ extrusion and for protection against apoptosis in embryonic cardiomyocytes (8). NCX2 expression is restricted to the CNS, brain, and spinal cord (9). Evidence for an essential role of NCX2 in the clearance of residual $\mathrm{Ca}^{2+}$ in presynaptic terminals during hippocampal short- and long-term plasticity was recently reported (9). The expression of the NCX3 gene is restricted to brain and skeletal muscle (5-8).

In skeletal muscle fibers, the presence of NCX1 and NCX3 has been detected on the sarcolemma, but the exact roles played by these exchangers are still unclear $(7,8,10)$. The expression of both exchanger proteins is developmentally regulated in rat skeletal myotubes, and their different subcellular distribution might be associated with specific physiological functions $(8,10,11)$. 
In the present study, we report the production and the analysis of $N c \times 3^{-/-}$mice. Using histological analysis, electromyography (EMG), and electrophysiological techniques as well as behavior testing, we show that $N c \times 3^{-/-}$mice have localized muscle fiber necrosis and inflammatory infiltrates, evidence of defective transmission at the neuromuscular junction (NMJ) as well as fatigability of limb muscles. Our results provide a basis for a better understanding of the physiological role of NCX3 in the muscle fiber and at the NMJ.

\section{Methods}

Generation of Ncx3 knockout mice. Exon 2 of the murine Ncx3 gene (GenBank, accession number AF321404), which encodes for the first two-thirds of the protein, was cloned from a 129/Sv genomic phage library. A targeting vector was constructed by inserting the neomycin resistance cassette (neo) into the unique EcoRI restriction site of that exon. Standard procedures were used to generate $N c x 3^{+/-}$mice. Experimental mice were 2 to 6 months old, of both sexes, and had F2 genetic backgrounds from 129/Sv and CD1 mice. Control mice consisted of age-matched littermates with two WT alleles at the $N c \times 3$ locus $\left(N c \times 3^{+/+}\right)$. All animal studies were authorized by the Animal Care Use and Review Committee of the Université Libre de Bruxelles.

RNA bybridization and RT-PCR. Messenger RNA (0.5 $\mu \mathrm{g}$, MicrofastTrack 2.0 kit, Invitrogen, Carlsbad, California, USA) was extracted from gastrocnemius muscles. The RNA probe was an ${ }^{\alpha 32} \mathrm{P}[\mathrm{UTP}]$-radiolabeled 450 bp in vitro-transcribed DNA fragment (MAXIscript, Ambion, Austin, Texas, USA). A GAPDH riboprobe was used to normalize the amount of RNA present on the membrane. OneStep RT-PCR was performed according to the manufacturer's protocol (Qiagen, Valencia, California, USA). The primers pair was as follows: $N c x 3,5^{\prime}$ CACTGTGGTTCTGAAGCCAGG-3' (located in exon 2) and $5^{\prime}$-TTGGGGTGTTCACCCAATACT-3' (located in exon 9); hypoxanthine phosphoribosyltransferase (HPRT) served as a standard to balance the amount of RNA used, 5'-GCTGGTGAAAAGGACCTCT- $3^{\prime}$ and $5^{\prime}$-CACAGGACTAGAACACCTGC-3.

Primary antibodies. (i) Anti-mouse NCX3 exchanger; peptide DGNFTPLEGKEVDES from the mouse NCX3 protein sequence was used for rabbit immunization (Eurogentec, Herstal, Belgium). This antibody was affinity purified and used at the dilution of $1: 1000$ for immunoblots, or 1:30 for immunocytochemistry. No cross-reaction was observed with NCX1. (ii) Anti-vesicular acetylcholine transporter (anti-VAChT): rabbit anti-serum (Phoenix, Belmont, California, USA) was used at 1:1000. $\alpha$-Bungarotoxin conjugated with Alexa Fluor 647 (Molecular Probes, Eugene, Oregon, USA) was used at 1:50. (iii) Anti-NCX1 and anti-plasma membrane calcium ATPase type 1 (anti-PMCA1): rabbit anti-sera anti-NCX1 and anti-PMCA1 (Swant, Bellinzona, Switzerland) were used at 1:1,000. (iv) Antidesmin: mouse anti-desmin monoclonal antibody (Molecular Probes) was used at 1:100.
Secondary antibodies. (i) Peroxidase-conjugated goat anti-rabbit (Biosource International, Camarillo, California, USA) and sheep anti-mouse (Amersham, Buckinghamshire, United Kingdom) were used at the dilution of 1:25,000 for immunoblots. (ii) FITC or Texas Re-conjugated donkey anti-rabbit were used at the dilution of 1:200 (Jackson Immunoresearch Laboratories, West Grove, Pennsylvania, USA).

Membrane preparation and immunoblot analysis. A supernatant containing membrane proteins of skeletal muscle homogenates was analyzed by SDS/PAGE. Detection by ECL Plus detection reagents (Amersham) was performed as described by the manufacturer. At least two different muscle homogenates were analyzed for each immunodetection. Protein loading was determined with a mouse anti-desmin monoclonal antibody (Molecular Probes).

Measurements of cytosolic $\left[\mathrm{Ca}^{2+}\right]_{i}$. Fibers from flexor digitorum brevis (FDB) muscles were isolated, and loaded with $1 \mu \mathrm{M}$ Fura-PE3/AM (Calbiochem, San Diego, California, USA) according to De Backer et al. (12). Solutions used were previously described (13). To stimulate $\mathrm{Ca}^{2+}$ influx via $\mathrm{Na} / \mathrm{Ca}$ exchange, all added $\mathrm{Na}^{+}$to the solution was replaced with $N$-methyl-D-glucamine (NMDG) (13).

Histological and histochemical analysis. Gastrocnemius muscle cryosections ( $15 \mu \mathrm{m}$ thick) were prepared according to Vanderwinden et al. (14) and processed for H\&E staining.

Immunofluorescence. Double or triple immunofluorescence was performed according to Vanderwinden et al. (15). A minimum of two different preparations was used for each combination of markers. In some immunofluorescence experiments, nuclei were stained with $5 \mu \mathrm{M}$ propidium iodide (Molecular Probes) in Tris$\mathrm{HCl} 0.05 \mathrm{M}$ (pH 7.4), containing $0.5 \mathrm{mg} / \mathrm{ml}$ ribonuclease A, for 2 hours in the dark at room temperature.

Confocal microscopy. Optical sections through regions of interest were collected sequentially for each fluorochrome as described (15).

Evans blue injection. Evans blue dye (EBD; SigmaAldrich, St. Louis, Missouri, USA; 10 mg/ml PBS, 50 $\mu \mathrm{l} / 10 \mathrm{~g}$ body weight) was injected into the retro-orbital vein; 12 to 24 hours after injection, mice were killed, skinned, and visually inspected for blue coloration as described (16). Cryosections of gastrocnemius muscle were examined by confocal microscopy.

Electrophysiological investigations. Mice were anesthetized with chloral hydrate (loading dose: $0.4 \mathrm{mg} / \mathrm{kg}$ of body weight intraperitoneally, followed by administration of $0.4 \mu \mathrm{g} / \mathrm{min}$ using a microinjection pump) and fixed on a table (17). The sciatic nerve and the gastrocnemius were exposed as described (17). The stimulating cathode was a steel needle electrode inserted close to the proximal portion of the sciatic nerve at a distance of about 3 $\mathrm{mm}$ from the reference electrode (anode). EMG responses were amplified, filtered, and displayed using a NeuroMax 4 device (Xltek, Oakville, Ontario, Canada).

Recruitment curve. The sciatic nerve was stimulated at increasing intensities and the peak-to-peak amplitude of the compound muscle action potential (CMAP) of the gastrocnemius muscle was measured. The mean 


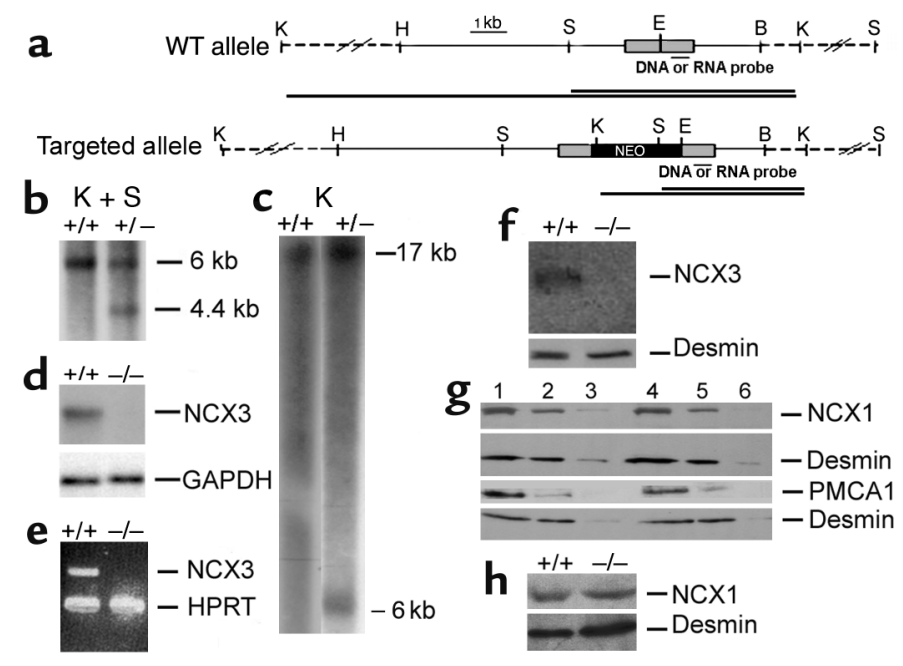

Figure 1

Targeted disruption of the Ncx3 gene. (a) Structure of the WT and the targeted alleles. The second exon (shaded boxes), the neomycin resistance cassette (black box), the probes used in DNA and RNA hybridization analysis (black bars underneath the second exon), as well as the DNA fragments generated after digestion with Knpl + Spel or Kpnl alone are represented. K, Kpnl; H, HindIII; S, Spel; E, EcoRI; B, BamHI. (b and $\mathbf{c}$ ) DNA hybridization analysis of Kpnl + Spel- (b) and Kpnl- (c) digested genomic DNA isolated from ES clones using the depicted probe. (d) RNA hybridization analysis. Messenger RNA $(0.5 \mu \mathrm{g} /$ lane) was hybridized with Ncx3 or GAPDH RNA probes. (e) RT-PCR analysis. Ncx3 amplicon ( $600 \mathrm{bp})$ and housekeeping gene HPRT amplicon ( $250 \mathrm{bp})$ were simultaneously amplified from gastrocnemius muscle messenger RNA by one-step RT-PCR. (f, $\mathbf{g}$, and $\mathbf{h}$ ) Western blot analysis. (f) Membrane fractions ( $50 \mu \mathrm{g} /$ lane) from gastrocnemius muscle were analyzed with anti-NCX3-specific antibody. (g) Immunodetection of membrane fractions from $\mathrm{Ncx}^{+/+}$(lanes $\left.1-3\right)$ and $\mathrm{Ncx} 3^{-/-}$(lanes 4-6) gastrocnemius muscle analyzed with anti-NCX1- and anti-PMCA1-specific antibodies. Amount of protein loaded: lanes 1 and 4, 50 $\mu \mathrm{g}$; lanes 2 and 5, $15 \mu \mathrm{g}$; lanes 3 and 6, $5 \mu \mathrm{g}$. (h) Membrane fractions $(20 \mu \mathrm{g} /$ lane) from FDB muscle were analyzed with anti-NCX1-specific antibody. Protein loading in $(\mathbf{f}-\mathbf{h})$ was determined by using a mouse anti-desmin monoclonal antibody.

CMAP was calculated at intensities from 0.05 to $10 \mathrm{~mA}$ (steps of $0.01 \mathrm{~mA}$ until maximal response, followed by steps of $0.5 \mathrm{~mA}$ ) using a Digitimer DS7 stimulator (Digitimer, Hertfordshire, United Kingdom). Filters were set at $30 \mathrm{~Hz}-1.5 \mathrm{KHz}$.

Repetitive nerve stimulations. The CMAPs were recorded in response to direct supramaximal stimuli applied to the sciatic nerve (18). During each test, five stimuli were given, and this procedure was repeated twice (filters: $30 \mathrm{~Hz}-1 \mathrm{KHz}$ ). The fractional decrease (decrement) or increase (increment) in amplitude of the CMAP was calculated using the peak-to-peak amplitude of the first and fifth responses. The results of three test series were averaged and a positive decremental or incremental response was defined as a decrease or an increase of more than $10 \%$ of the fifth compared to the first response (19).

Postexercise facilitation. The testing program was the following: (i) a single repetitive stimulation at $10 \mathrm{~Hz}$ was recorded at rest $\left(t_{0}\right)$; (ii) the sciatic nerve was electrically stimulated at $10 \mathrm{~Hz}$ for 20 minutes (exercise); (iii) CMAPs were recorded after $10-\mathrm{Hz}$ repetitive stimulations, immediately after exercise $\left(t_{20}\right)$, and every 30 seconds $\left(t_{20+n}\right)$ for 5 minutes; (iv) after a resting period of 30 minutes, the same animals were tested with the same program at $30-\mathrm{Hz}$ stimulation.

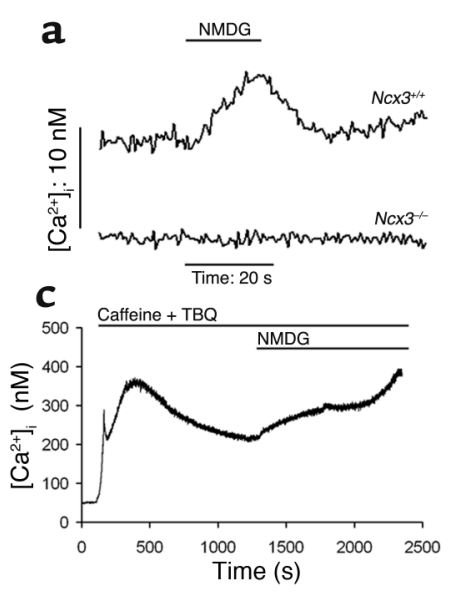

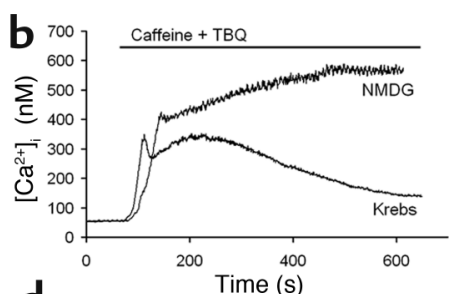

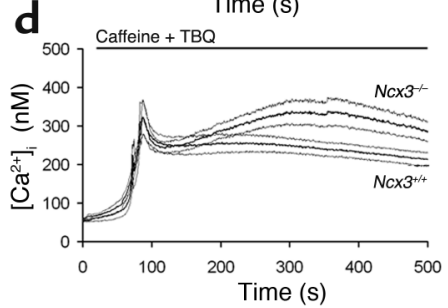

\section{Figure 2}

NCX activity in FDB muscle. [Ca $\left.{ }^{2+}\right]$ i was measured in FDB fibers loaded with Fura-PE3. (a) Response to extracellular $\mathrm{Na}^{+}$removal (replaced by NMDG). Graphs are representative of $\left[\mathrm{Ca}^{2+}\right]_{i}$ transients from $\mathrm{Ncx} 3^{+/+}(n=7)$ and $\mathrm{Ncx3}^{-/-}$ $(n=9)$ FDB fibers. (b) Response to caffeine and TBQ in the presence (Krebs) or in the absence of extracellular $\mathrm{Na}^{+}$. Graphs are representative of five experiments with $\mathrm{Nc \times 3} 3^{+/+}$ FDB fibers. (c) Response to caffeine and TBQ in $\mathrm{Ncx3}^{-/-}$ fibers in the absence of extracellular $\mathrm{Na}^{+}$. The graph is representative of five experiments. (d) Comparison of the $\mathrm{Na} / \mathrm{Ca}$ exchange activity in response to caffeine and TBQ in $\mathrm{Ncx}^{+/+}(n=7)$ and $\mathrm{Ncx}^{-/-}(n=7)$ FDB fibers. Results are displayed as the mean (bold traces) \pm SEM (thin traces). 
Stimulated single-fiber electromyography. The cathode was inserted close to the proximal sciatic nerve, and a stimulated single-fiber electromyography (SFEMG) electrode (Medtronic 26G-40 mm, core diameter: $25 \mu \mathrm{m}$; Medtronic, Minneapolis, Minnesota, USA) was introduced into the gastrocnemius muscle using a micromanipulator. Single-fiber action potentials (SFAPs) were recorded using dedicated software at filter settings of $500 \mathrm{~Hz}-10$ $\mathrm{KHz}$. Amplifier gain was $200 \mu \mathrm{V}-1 \mathrm{mV} /$ division adjustable. Adequate current flow typically ranged from 0.2 to $2.7 \mathrm{~mA}$. Single fibers were selected as described (20). For each fiber, one response corresponds to 50 consecutive SFAPs. These measures were repeated one to four times to calculate the mean consecutive discharge (MCD) of the potential latencies (17). In order to assess the relationship between MCD values and increasing discharge rates (21-23), stimuli were delivered successively at 10 and 20 $\mathrm{Hz}$ (WPI A310-A365 stimulator; World Precision Instruments, Hertfordshire, United Kingdom).

In vitro microelectrophysiology. Hemidiaphragm muscles with their associated phrenic nerves were isolated, and electrophysiological recordings were done according to Minic at al. (24). Evoked endplate potentials (EPPs) and/or spontaneous miniature endplate potentials (MEPPs) were recorded with intracellular microelectrodes filled with $3 \mathrm{M} \mathrm{KCl}$ solution (8-12 $\mathrm{M} \Omega$ resistance) using conventional techniques (24). The motor nerve was stimulated via a suction electrode with current pulses of 0.1ms duration and supramaximal voltage (typically 3-8 V) at different stimulating frequencies. Synaptic potentials were recorded in about four to six NMJs chosen randomly in each preparation. If necessary, $\mu$-conotoxin GIIIB was used to prevent muscle contraction. The EPP amplitudes recorded on junctions treated with $\mu$-conotoxin GIIIB were normalized to $-75 \mathrm{mV}$ membrane potential and corrected for nonlinear summation $(25,26)$. The quantal content of EPPs (m) was calculated at each junction by dividing the mean normalized and corrected EPP amplitude by the mean normalized MEPP amplitude.

Twitch tension measurements. Contractile responses of isolated mouse hemidiaphragms were elicited by stimulating the phrenic nerve with supramaximal current pulses (0.15-ms duration) at various frequencies (20, 40, 60, 80, and $100 \mathrm{~Hz}$ ), as previously described (27).

Endurance and coordination analyses. Mice were tested blindly using littermate controls. The wire test was based on the taut wire test used by Gomez (18). For testing, mice were placed with front claws gripping the wire and observed for a period of 180 seconds of suspension. The chronograph was stopped any time the animal fell or reached a rest area and restarted when the animal was placed again on the wire. Number of successes (when an animal reached a rest area) and number of falls from the wire were recorded. The average scores for successes and falls were calculated as described (28). Each animal was tested only once. The Rota-Rod (7600; Ugo Basile, Comerio, Italy) rotated at a constant speed of $32 \mathrm{rpm}$. The mice were acclimatized to the treadmill by being placed on it three times the day before the experiment. Each mouse was placed on the rotating bar, and the duration the mouse remained on the bar was measured, up to a maximum of $300 \mathrm{sec}-$ onds. Three consecutive sessions were conducted. After a rest period of 1 hour, the same mice were tested for endurance over the course of 2,000-second maximums.

Statistics. The mean \pm SD and mean \pm SEM were calculated using a statistical program (GraphPad Instat; GraphPad Software, San Diego, California, USA). A Student's $t$ test was used for simple comparison of means. For multiple comparisons, data were analyzed by ANOVA, followed by the Bonferroni multiple comparisons procedure.

\section{Results}

Generation of Ncx3-deficient mice. A mutant allele was constructed as described in Methods (Figure 1a). After electroporation with the targeting vector, the recombinant embryonic stem (ES) clones were identified by DNA hybridization (Figure 1, b and c) and used to produce chimeric mice. Transmission of the mutant allele produced $N c x 3$ heterozygous mice that were mated to generate $N C X 3$ null mutants $\left(N c x 3^{-/-}\right)$. Ncx $3^{-/-}$mice were viable and fertile; they appeared grossly normal and showed normal body weight (data not shown). The absence of NCX3 messenger RNA in hybridization assay and in RT-PCR (Figure 1, $\mathrm{d}$ and e), as well as the absence of NCX3 immunoreactivity (NCX3-ir) (Figure 1f) in $\mathrm{Ncx3}^{-/-}$gastrocnemius muscle, confirmed that the insertion of the neomycin resistance cassette in the second exon of the Ncx3 gene created a null mutation.
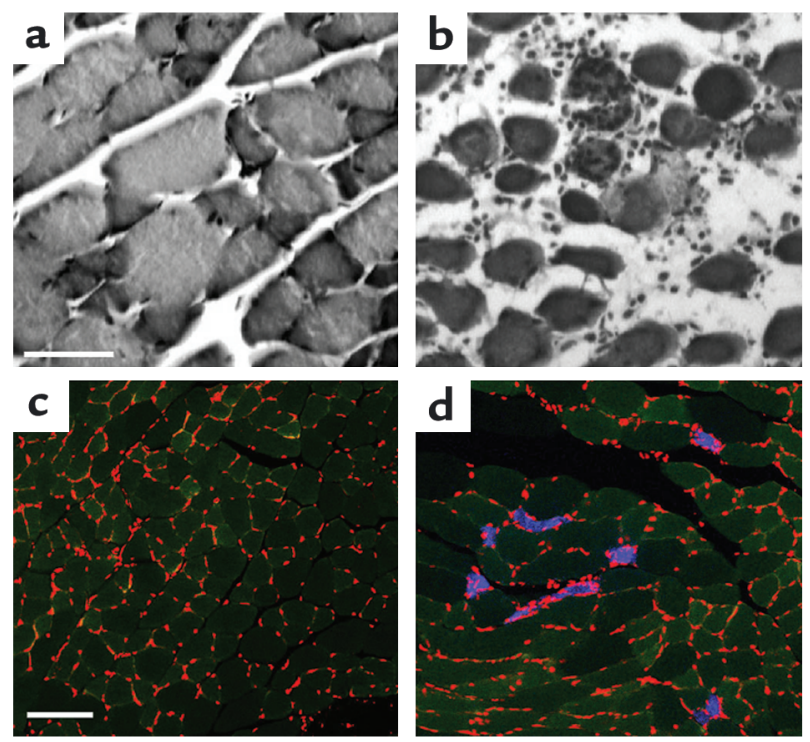

\section{Figure 3}

Histological alterations in $N c \times 3^{-/-}$muscles. H\&E-stained sections from 3-month-old $\mathrm{Ncx}^{+/+}$(a) and $\mathrm{Ncx}^{-/-}$(b) gastrocnemius muscle: focus of fiber necrosis and cellular infiltrate in $\mathrm{Ncx}^{-/-}$mice. Scale bar: $50 \mu \mathrm{m}$. Localization of EBD in cryosections of gastrocnemius muscles: 3-month-old EBD-injected $\mathrm{Ncx3}^{+/+}(\mathbf{c})$ and $\mathrm{Ncx}^{-/-}$(d) mice were examined after 12-24 hours. Red stained structures are nuclei, green stained areas are cytosol, and blue stained areas are EBD-positive fibers. Scale bar: $100 \mu \mathrm{m}$. 

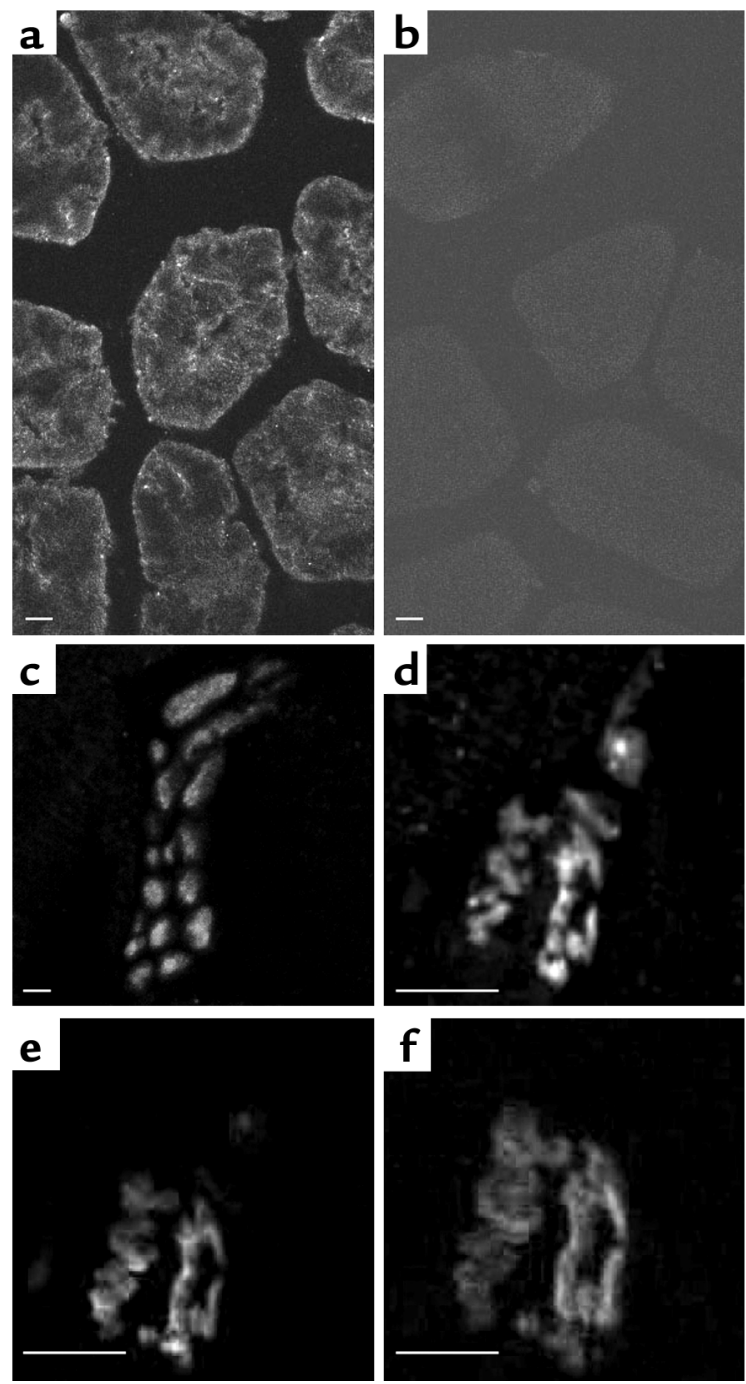

Figure 4

Immunolocalization of NCX3 in sections of $\mathrm{Ncx}^{+/+}$and $\mathrm{Ncx}^{-/-}$gastrocnemius muscles. ( $\mathbf{a}$ and $\mathbf{b}$ ) Both sarcolemma and sarcoplasm from $\mathrm{Ncx}^{+/+}$fibers are labeled with a polyclonal anti-NCX3 antibody (a); no immunoreactivity is detected in $\mathrm{Ncx}^{-/-}$fibers (b). (c) Nerve ramifications inside the gastrocnemius muscle are also NCX3 positive. (d-f) Detection of NCX3 protein at the NMJ: triple labeling experiment with anti-NCX3 antibody (d), anti-VAChT antibody (e), and with the Alexa fluor 647- $\alpha$-bungarotoxin (f) in a transverse section $(15 \mathrm{~mm})$ of $\mathrm{Ncx}^{+/+}$gastrocnemius muscle. Scale bars: $10 \mathrm{~mm}$.

Absence of compensatory overexpression of NCX1 and PMCA1 proteins in Ncx3 $3^{-/-}$gastrocnemius muscles. Since NCX1 and PMCA1 are also involved in the control of $\mathrm{Ca}^{2+}$ homeostasis and well expressed in skeletal muscles $(10,29)$, their expression in membrane protein extracts isolated from $\mathrm{Ncx}^{+/+}$and $\mathrm{Ncx} 3^{-/-}$gastrocnemius muscle was analyzed by Western blotting. No compensatory overexpression was detected in $\mathrm{Nc} 3^{-/}$gastrocnemius muscles: NCX1- and PMCA1-ir's were very similar in both genotypes (Figure $1 \mathrm{~g})$. Similar results were obtained when analyzing the presence of NCX1 in $\mathrm{Ncx}^{+/+}$and $\mathrm{Ncx} 3^{-/-}$ FDB fibers (Figure 1h).
Reduction of $\mathrm{Na} / \mathrm{Ca}$ exchange activity in $\mathrm{Ncx3^{-/- }}$ FDB fibers. In order to study $\mathrm{Na} / \mathrm{Ca}$ exchange activity in muscle fibers, we examined $\mathrm{Ca}^{2+}$ signaling in FDB fibers. In $\mathrm{Ncx}^{+/+} \mathrm{FDB}$ fibers, a typical response to the superfusion of low-Na medium (where $\mathrm{Na}^{+}$is replaced by NMDG), was observed: the withdrawal of extracellular $\mathrm{Na}^{+}$was followed by a rise of about $7 \pm 3 \mathrm{nM}($ mean $\pm \mathrm{SEM}, n=6)$ in $\left[\mathrm{Ca}^{2+}\right]_{\mathrm{i}}$, reflecting the reverse $\mathrm{Na} / \mathrm{Ca}$ exchange activity (Figure $2 \mathrm{a}$ ). When $\mathrm{Na}^{+}$was reintroduced into the bath, basal $\left[\mathrm{Ca}^{2+}\right]_{i}$ was restored, illustrating forward exchange activity. In $\mathrm{Ncx} 3^{-/}$ FDB fiber, no reverse exchanger activity was observed under these conditions: $\left[\mathrm{Ca}^{2+}\right]_{\text {i }}$ remained unchanged when the low- $\mathrm{Na}^{+}$medium was superfused (Figure 2a). We then studied the forward mode of the exchanger after elevating $\left[\mathrm{Ca}^{2+}\right]$ i with $15 \mathrm{mM}$ caffeine, which stimulates sarcoplasmic reticulum (SR) $\mathrm{Ca}^{2+}$ release, and $1 \mu \mathrm{M}$ 2,5-di(tertbutyl)-1,4-benzohydroquinone (TBQ), which inhibits SR $\mathrm{Ca}^{2+}$ ATPases (SERCAs) (Figure 2b). As expected (13), this treatment produced a transient peak in $\left[\mathrm{Ca}^{2+}\right]_{\mathrm{i}}$ followed by a very slow decline. This second phase was inhibited when extracellular $\mathrm{Na}^{+}$was replaced by NMDG, suggesting that the decline was due to an efflux of $\mathrm{Ca}^{2+}$ through $\mathrm{Na} / \mathrm{Ca}$ exchange (Figure 2b). In contrast to the observation made at a basal $\left[\mathrm{Ca}^{2+}\right]_{i}$ where reverse $\mathrm{Na} / \mathrm{Ca}$ exchange activity was absent (Figure 2a), a forward $\mathrm{Na} / \mathrm{Ca}$ exchange activity was detected in $\mathrm{Ncx}^{-/-}$FDB fibers incubated with caffeine and TBQ (Figure 2c). Indeed, like in $\mathrm{Ncx3^{+/+ }}$ fibers, the secondary decline in $\left[\mathrm{Ca}^{2+}\right]_{\text {i }}$ present in $\mathrm{Nc} \times 3^{-/-}$fibers was also suppressed in the absence of extracellular $\mathrm{Na}^{+}$. However, the $\mathrm{Na} / \mathrm{Ca}$ exchange activity was significantly lower in $\mathrm{Ncx}^{3^{-/-}}$than in $\mathrm{Ncx}^{+/+}$FDB fibers (Figure 2d): treatment of FDB fibers with caffeine and TBQ induced a peak of similar magnitude in $\mathrm{Ncx}^{+/+}$and $\mathrm{Ncx}^{-/-}$cells, but the secondary phase was significantly slower in the latter $\left(\left[\mathrm{Ca}^{2+}\right]_{\mathrm{i}}\right.$ after $275 \mathrm{~s}: 252 \pm 20 \mathrm{nM}$ and $326 \pm 27 \mathrm{nM}$ in $N c \times 3^{+/+}$and $N c \times 3^{-/-}$mice, respectively; Student's $t$ test: $P<0.01, n=7$ in both groups). Basal $\left[\mathrm{Ca}^{2+}\right]_{\mathrm{i}}$ was not significantly different between control and mutant mice $\left(53 \pm 2 \mathrm{nM}\right.$, and $59 \pm 2 \mathrm{nM}$ in $N c \times 3^{+/+}$and $N c x 3^{-/-}$fibers, respectively; Student's $t$ test: $P>0.5, n=7$ in both groups). The data correspond to the mean \pm SEM.

Histological alterations in $\mathrm{Nc} \times 3^{-/-}$skeletal muscles. Crosssections of muscle from $N c \times 3^{+/+}$and $N c \times 3^{-/-}$mice were stained with H\&E and examined by light microscopy (Figure 3, a and b). Foci of necrotic fibers, representing less than $10 \%$ of the total fibers, associated with mononuclear infiltrates were observed in $\mathrm{Nc} 3^{-/-}$muscles (Figure $3 b$ ). The presence of damaged fibers in $\mathrm{Nc} 3^{-/-}$muscles was confirmed with EBD. After EBD injection, inspection revealed in four of six $\mathrm{Nc} 3^{-/-}$mice a streaky blue coloration of some skeletal muscles, with marked individual variation in both intensity and extent (data not shown). EBD uptake in muscle fibers was confirmed by confocal microscopy (Figure 3, c and d). In any given muscle, EBD-positive fibers occurred either singly or in small clusters, and their number varied from animal to animal but was always inferior to $10 \%$ of the total fibers. Conversely, in $N c \times 3^{+/+}$mice, no EBD uptake into skeletal muscles was observed by gross inspection $(n=6)$, 
and less than $0.1 \%$ of total fibers was shown to be EBD positive by microscopic observation (Figure 3c).

Immunolocalization of NCX3 in muscle and nerve fibers as well as at the neuromuscular junction. Immunofluorescence studies were performed on 3-month-old $\mathrm{Ncx}^{+/+}$and $\mathrm{Ncx}^{-/-}$ gastrocnemius cryosections. NCX3-ir was detected in all muscle fibers in $\mathrm{Ncx}^{+/+}$but not in $\mathrm{Ncx}^{-/-}$animals (Figure $4, a$ and $b$ ). In addition, NCX3-ir was also observed in nerve ramifications present between muscle fibers (Figure 4c). Confocal studies of cross-sectioned myofibers stained with NCX3 antiserum, VAChT antiserum (a marker for nerve terminals) and Alexa fluor 647- $\alpha$-bungarotoxin (a specific probe for nicotinic acetylcholine receptors) indicated the presence of NCX3-ir at the NMJ (Figure 4, d-f).

$E M G$ alterations in $\mathrm{Ncx}^{-1-}$ mice. An extensive EMG analysis was performed on $\mathrm{Ncx}^{+/+}$and $\mathrm{Nc} 3^{-/-}$mice. First, recruitment curve determination was performed. At increasing stimulus intensities, alterations in the recruitment curve recordings were detected: significantly reduced CMAPs were observed in $\mathrm{Ncx}^{-/-}$mice, as compared with $\mathrm{Ncx}^{+++}$mice (Figure 5a). The slope of the curve was also significantly reduced in the mutant mice $(P<0.05$ from 0 to $5 \mathrm{~mA}$ ). The latency of the muscle fiber responses was identical in both genotypes (data not shown). Second, responses evoked in the gastrocnemius muscle with repetitive nerve stimulation (RNS) at 10 and $30 \mathrm{~Hz}$ were analyzed (Figure 5, b and c). At a low rate of repetitive stim- ulation (LRRS), a decremental CMAP was recorded in $N c \times 3^{-/}$mice (mean decrement \pm SEM: $11.78 \pm 1.19 \%$ and $0.03 \pm 0.30 \%$ for $\mathrm{Ncx}^{-/-}(n=8)$ and $\mathrm{Ncx} 3^{+/+}$mice $(n=5)$, respectively; Student's $t$ test: $P<0.001)$. Unexpectedly, at high rate of repetitive stimulation (HRRS), an increment was recorded in four of eight $\mathrm{Ncx}^{-/-}$mice. The mean increment at HRRS was $51.69 \pm 2.76 \%$ in these four $\mathrm{Ncx} 3^{-/-}$mice, as compared with a decrement of $13.57 \pm$ $0.28 \%$ in $\mathrm{Ncx}^{+/+}$mice $(n=5)$. In order to improve the sensitivity of our investigations, we analyzed the postexercise facilitation (21). At LRRS, the decrease of the CMAP was significantly more profound in the knockout group immediately after the 20-minute exercise period (Figure 5d). In contrast, a marked increment was observed at HRRS in $\mathrm{Ncx}^{-/-}$mice (Figure 5e). The recovery was quicker in the control group whatever the frequency. The ratio between the percent of change recorded at HRRS divided by the percent of change recorded at LRRS showed the prominent postexercise facilitation (Figure 5f). Finally, stimulated SFEMG was performed in $\mathrm{Ncx}^{+/+}$and $\mathrm{Ncx}^{-/-}$ mice. SFAPs were recorded following stimulation, and the MCDs of the potential latencies were calculated. Sixtyseven responses were recorded from $23 \mathrm{Ncx}^{-/-}$single fibers, and 88 responses from $22 \mathrm{Ncx}^{+/+}$single fibers. MCDs were significantly different between $\mathrm{Ncx}^{+/+}$(range: $30-84 \mu \mathrm{s} ;$ mean $\pm \mathrm{SEM}=58.8 \pm 0.6 \mu \mathrm{s})$ and $\mathrm{Nc} 3^{-/-}$mice (range: $47-130 \mu \mathrm{s}$; mean $\pm \mathrm{SEM}=82.3 \pm 0.8 \mu \mathrm{s}$; intergroup a

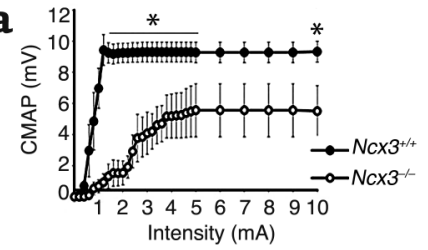

b
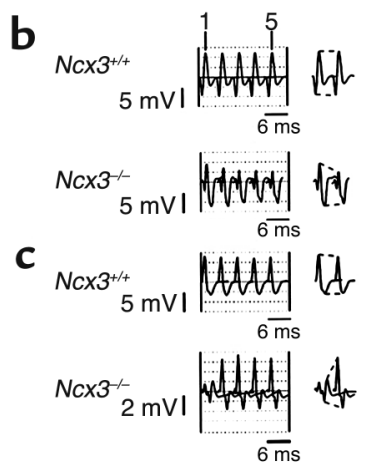

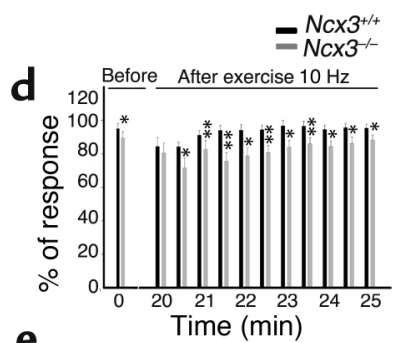

e

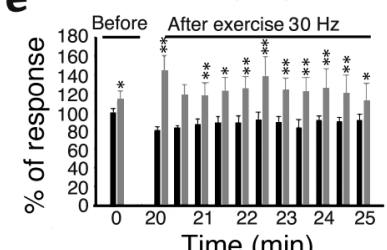

$f$

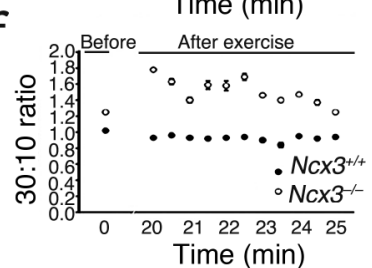

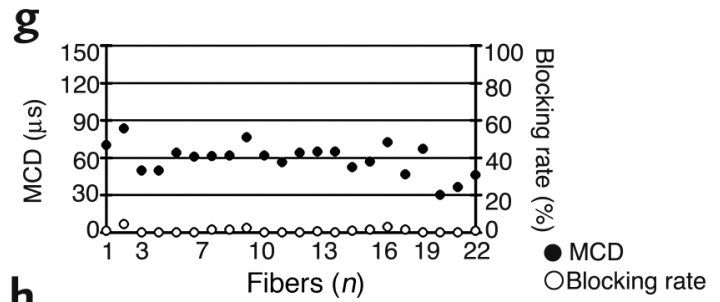

h

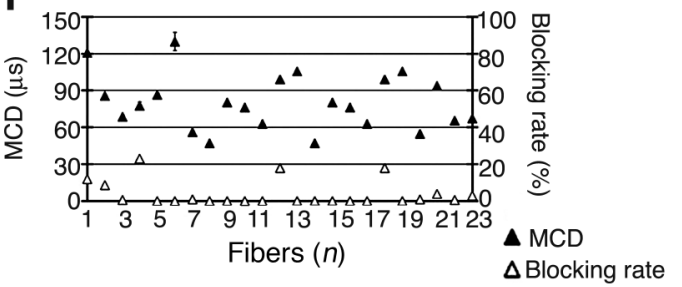

Figure 5

Electromyographic findings in $\mathrm{Ncx}^{-/-}$mice. (a) Recruitment curves of muscle responses evoked in the gastrocnemius muscle by stimulation of the sciatic nerve at increasing intensities. Mean CMAPs obtained in $\mathrm{Ncx}^{+/+}(n=5$; filled circles $)$ and $N c x 3^{-/-}(n=5$; open circles $)$ mice are illustrated. (b and $\mathbf{c}$ ) Effects of repetitive stimulation of the sciatic nerve at 10 (b) and $30 \mathrm{~Hz}(\mathbf{c})$ in $\mathrm{Ncx}^{+/+}$and $\mathrm{Ncx} 3^{-/-}$mice. The graphs shown are representative of all the mice tested in the same group, except for $\mathrm{Ncx}^{-/-}$mice at HRRS, where the graph is representative of one out of the 4/8 mice with an incremental CMAP. (d and e) Time course of the fifth response amplitude/first response amplitude ratios during LRRS (d) and HRRS (e) before and after exercise (20 minutes of repetitive stimuli) in $\mathrm{Ncx}^{+/+}\left(n=5\right.$; black bars) and $\mathrm{Ncx}^{-/-}(n=5$; gray bars) mice. (f) Ratios of the values illustrated in (d) and (e) (filled circles, $N_{c x} 3^{+/+}$mice; open circles, $N_{c x} 3^{-/-}$mice). (g and h) MCDs expressed in microseconds and blocking rates expressed in percent in $N c \times 3^{+/+}$mice $(n=22$ fibers; MCDs, filled circles; blocking rate, open circles) and $N c \times 3^{-/-}$mice $(n=23$ fibers; filled triangles, MCDs; open triangles, blocking rate). Statistics (in a, $\mathbf{d}-\mathbf{h})$ : mean $\pm \mathrm{SEM} ;{ }^{*} P \leq 0.05 ;{ }^{*} P \leq 0.01$. 

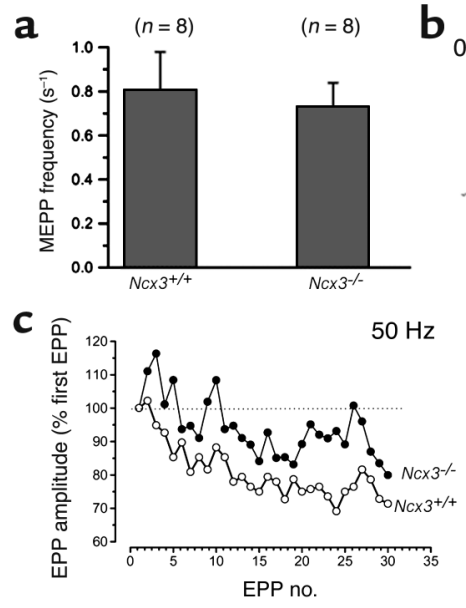
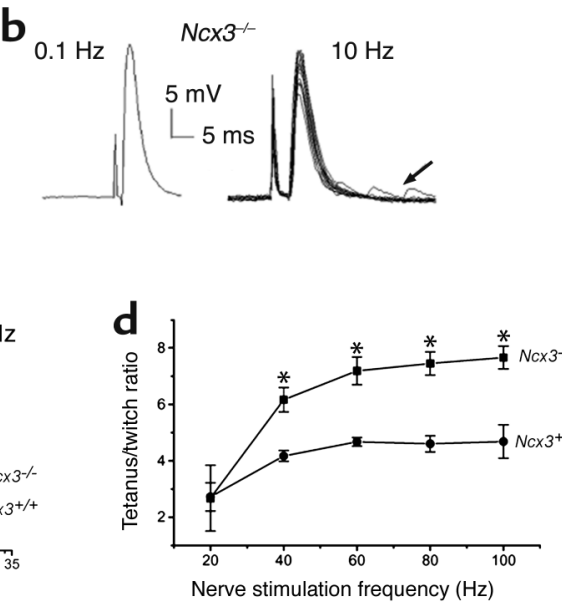

\section{Figure 6}

Synaptic parameters recorded in hemidiaphragm neuromuscular preparations from $\mathrm{Ncx}^{+/+}$and $\mathrm{Ncx}^{-/-}$mice. (a) Spontaneous quantal ACh release, measured as the frequency of MEPPs. The number of NMJs tested in each group is indicated in brackets. (b) Full-size EPP evoked at $0.1 \mathrm{~Hz}$ by a single nerve stimulus in a $\mathrm{Ncx}^{-/-} \mathrm{NMJ}$ (left tracing) and after repetitive stimulation at $10 \mathrm{~Hz}$ (right tracing). The arrow indicates the increased MEPP frequency. Standard physiological solution supplemented with $2.2 \mu \mathrm{M} \mu$-conotoxin GIIIB was used. The resting membrane potential during measurements was $-74 \pm 0.2$ $\mathrm{mV}$. (c) EPP amplitudes evoked at $50-\mathrm{Hz}$ nerve stimulation (expressed as percent of the first EPP of each train, in sequential order). (d) Nerve-evoked, tetanus-twitch ratio at different stimulation frequencies in hemidiaphragm muscles from $\mathrm{Ncx}^{+/+}$and $\mathrm{Ncx}^{-/-}$mice $(n=3)$. The resting membrane potential during recordings was $-72 \mathrm{mV}$. Mean $\pm \mathrm{SD}(\mathbf{a}$ and $\mathbf{d}) .{ }^{*} P \leq 0.001$. difference, $P<0.01$ ) (Figure 5, $\mathrm{g}$ and $\mathrm{h}$ ). Moreover, although 5 of $23 \mathrm{Ncx}^{--}$fibers presented an abnormal neuromuscular blocking (blocking rate ranged from 6 to $25 \%$ ), none of the $\mathrm{Ncx}^{+/+}$fibers showed a blocking rate higher than $5 \%$ (Figure 5, $\mathrm{g}$ and $\mathrm{h}$ ). In order to assess the relationship between MCD values and increasing firing rates (21-23), 21 fibers from $\mathrm{Ncx}^{-/-}$mice and 9 fibers from $\mathrm{Ncx}^{+/+}$mice were stimulated successively at 10 and $20 \mathrm{~Hz}$, respectively. A significant decrease of the mean MCDs from 77.2 to $64.4 \mu$ s was observed in $\mathrm{Ncx}^{-1-}$ fibers when stimulated at 10 and $20 \mathrm{~Hz}$, respectively. By contrast, at the same frequencies, the mean MCDs increased from 43.6 to $75.9 \mu \mathrm{s}$ in $\mathrm{Ncx}^{+/ /}$fibers (ANOVA, $P<0.01$, genotype X frequency).

Electrophysiological alterations in isolated $\mathrm{Ncx}^{-/-}$neuromuscular preparations. The NMJ of $\mathrm{Ncx}^{-1-}$ mice was explored with electrophysiological techniques in order to detect eventual changes in synaptic parameters. Spontaneous quantal transmitter release, measured as the frequency of MEPPs in a standard physiological solution, did not differ significantly in $\mathrm{Ncx}^{+/+}$and $\mathrm{Nc}^{-3^{-/-}} \mathrm{NMJs}(n=8$, Student's $t$ test: $P>0.3$ ) (Figure 6a). In $N c \times 3^{+/+}$junctions, the mean amplitude of MEPPs was $0.86 \pm 0.11 \mathrm{mV}(n=8)$, whereas in $\mathrm{Ncx}^{-/-}$muscles it was $0.95 \pm 0.16 \mathrm{mV}(n=8$, four different muscles; Student's $t$ test: $P>0.2$ ).

To test the eventual presynaptic effects of the absence of NCX3, the quantal content of EPPs was determined in $\mathrm{Ncx}^{3^{+/+}}$and $\mathrm{Ncx}^{-/-} \mathrm{NMJs}$. In isolated hemidiaphragms equilibrated in standard physiological solution supplemented with $2.2 \mu \mathrm{M} \mu$-conotoxin GIIIB, nerve stimulation at a low rate $(0.1 \mathrm{~Hz})$ evoked full-sized EPPs (Figure $6 \mathrm{~b}$, left panel). In $\mathrm{Ncx}^{--}$junctions, the mean amplitude of these EPPs was $25.7 \pm 1.8 \mathrm{mV}(n=4)$, and their mean quantal content was $32.6 \pm 2.5(n=4)$. These values did not differ significantly from $\mathrm{Ncx} 3^{+/+}$junctions (Student's $t$ test: $P>0.1)$. Thus, under the conditions tested, the basic quantal release parameters were not significantly different in $\mathrm{Ncx}^{+/+}$and $\mathrm{Ncx}^{-/-} \mathrm{NMJs}$. However, further experiments in $\mathrm{Ncx}^{-/-} \mathrm{NMJs}$ revealed that EPP amplitudes during continuous stimulation depended on nerve stimulation frequency and pulse number in a given train.
Indeed, at $10 \mathrm{~Hz}$ nerve stimulation, there was an initial decrease of EPP amplitudes that attained a plateau over an expanded time frame (Figure 6b, right panel). When analyzed as a function of time or pulse number, the decline of EPP amplitudes was found to be greater at 20 $\mathrm{Hz}$ than at $50 \mathrm{~Hz}$ stimulation frequency (data not shown). These results are in marked contrast to $\mathrm{Ncx}^{+/+} \mathrm{NMJs}$, in which the decline of EPP amplitudes was greater at high than at low nerve stimulation frequencies. A comparison of EPP amplitudes evoked at $50 \mathrm{~Hz}$ in $\mathrm{Ncx}^{+/+}$and $\mathrm{Ncx}^{-/-}$ NMJs also revealed that EPPs were initially slightly facilitated and thereafter depressed in the $\mathrm{Nc} 3^{+/+} \mathrm{NMJ}$, whereas in the $\mathrm{Ncx}^{-/-} \mathrm{NMJ}$, facilitation was more marked and lasted longer; several peaks of facilitation were observed superimposed to the EPP depression. These results indicate that transmitter mobilization and synaptic vesicle turnover were differently affected in $\mathrm{Ncx}^{-/-}$and $\mathrm{Ncx}^{+/+}$ nerve terminals following high-frequency stimulation. This was further substantiated by the finding that delayed transmitter release, measured as the number of quantal releases in the $100 \mathrm{~ms}$ after a train of nerve stimulation at $50 \mathrm{~Hz}$, was significantly and markedly increased in $\mathrm{Ncx}^{-/-}$ NMJs $(11.4 \pm 1.8 \mathrm{~Hz} ; n=3)$ when compared with $\mathrm{Ncx}^{+/+}$ NMJs $(2.2 \pm 0.4 \mathrm{~Hz} ; n=3$; Student's $t$ test: $P<0.001)$. The data correspond to the mean \pm SD.

Increased tetanus/contraction ratio at high-frequency nerve stimulation in $\mathrm{Ncx}^{3--}$ nerve-muscle preparations. Analysis of contractile responses of isolated hemidiaphragms elicited by phrenic nerve stimulation indicated that both $\mathrm{Ncx}^{+/+}$and $\mathrm{Ncx}^{3-/-}$ nerve-muscle preparations can maintain nerve-evoked tetanic contraction at frequencies between 40 and $100 \mathrm{~Hz}$, respectively, for 1 second without decline. However, the tetanus-twitch ratio was significantly higher in $\mathrm{Nc} 3^{-/-}$than in $\mathrm{Ncx}^{+/ /}$muscles (Figure 6d, $P<0.001 ; n=3$ ).

Decreased endurance and increased fatigue in $\mathrm{Nc} 3^{-/-}$mice. $\mathrm{Ncx} 3^{-/}$mice performed poorly in the wire and the RotaRod tests compared to $\mathrm{Ncx}^{+/+}$mice. Indeed, after 100 seconds spent on the wire, reduction in activity ("average success score": $1.4 \pm 0.4$ and $4.3 \pm 0.8$ for $\mathrm{Ncx}^{-/-}$and 

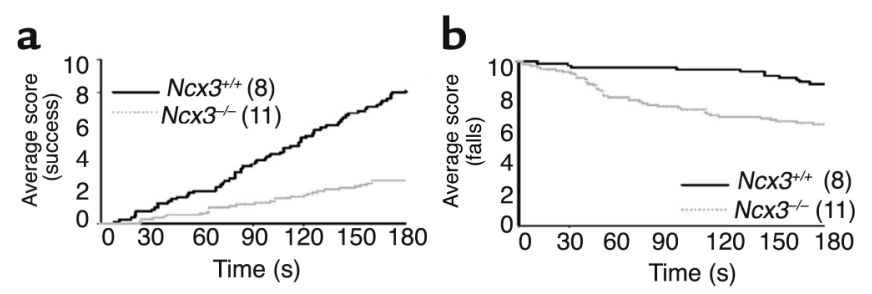

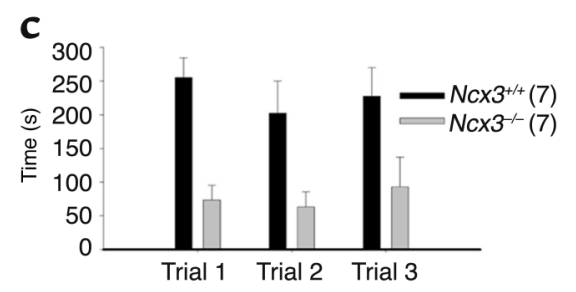

Figure 7

Behavior alterations in $\mathrm{Ncx}^{-/-}$mice. (a) Average success score and (b) average falling score of $\mathrm{Ncx}^{+/+}$and $\mathrm{Ncx3^{-/- }}$ mice in the wire test. (c) Time spent on the Rota-Rod. The number of animals used in each group is indicated in brackets. Mean \pm SEM (c).

$N c x 3^{+/+}$mice, respectively; Student's $t$ test: $\left.P<0.01\right)$ and weakness of forelimbs muscles ("average falling score": $7.1 \pm 0.7$ and $9.5 \pm 0.3$ for $\mathrm{Ncx}^{-/-}$and $\mathrm{Ncx} 3^{+/+}$mice, respectively; Student's $t$ test: $P<0.01)$ were significantly different between control and mutant groups (Figure 7, a and b). Whatever the Rota-Rod trial, $N\left(\mathrm{cx}^{---}\right.$mice stayed for a shorter mean duration on the rotating bar than $\mathrm{Nc} 3^{+/+}$mice (ANOVA, $P<0.001$, genotype $\mathrm{X}$ session) (Figure $7 \mathrm{c}$ ). Moreover, individual comparison of the

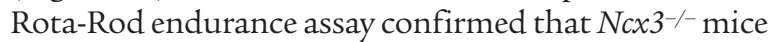
readily fatigue in comparison to their littermate controls: $\mathrm{Ncx}^{+++}$mice spent $20.3 \pm 4.5$ minutes on the RotaRod vs. $2.4 \pm 1.4$ minutes for $N c \times 3^{-/-}$mice (Student's $t$ test: $P<0.01)$. The data correspond to the mean \pm SEM.

\section{Discussion}

We have generated a mouse strain with null alleles in the gene coding for $\mathrm{NCX} 3$, the third isoform of the $\mathrm{Na} / \mathrm{Ca}$ exchanger, which is mainly expressed in brain and skeletal muscles (5). The absence of NCX3 mRNA in RNA hybridization and RT-PCR studies as well as of NCX3 protein in Western blotting and immunofluorescence analysis in skeletal muscles confirmed the null mutation in $\mathrm{Nc} 3^{-/-}$mice.

The absence of NCX3 in skeletal muscle fibers was associated with necrosis and cellular infiltrates. Our results indicated that necrosis was not the consequence of an increased basal $\left[\mathrm{Ca}^{2+}\right]_{\mathrm{i}}$. This was expected since the exchanger had a low affinity for $\mathrm{Ca}^{2+}\left(K_{\mathrm{m}} \sim 3 \mu \mathrm{M}\right)(30)$ and therefore had a minor influence on resting (low) $\left[\mathrm{Ca}^{2+}\right]_{\text {i }}$. On the contrary, the plasma membrane $\mathrm{Ca}^{2+}$ pumps had a higher affinity for $\mathrm{Ca}^{2+}$ but a lower maximal rate of transport and were therefore more suitable for regulating $\left[\mathrm{Ca}^{2+}\right]_{\mathrm{i}}$ in the low concentration range (e.g., at rest) (31). Balnave and Allen have shown that the forward-mode $\mathrm{Na} / \mathrm{Ca}$ exchange contributes to the removal of $\mathrm{Ca}^{2+}$ from fibers stimulated with TBQ and caffeine (13). This was confirmed here, and we showed that the absence of NCX3 results in a significantly lower $\mathrm{Na} / \mathrm{Ca}$ exchange activity and a prolonged secondary decline in $\left[\mathrm{Ca}^{2+}\right]_{i}$, emphasizing the high-transport capacity of this exchanger. The contribution of $\mathrm{Na} / \mathrm{Ca}$ exchange to $\mathrm{Ca}^{2+}$ efflux may become crucial in conditions of high and sustained $\left[\mathrm{Ca}^{2+}\right]_{i}$, as during fatiguing stimulation (32) or after repeated eccentric exercise (33). In these conditions, the absence of NCX3 could lead to altered $\mathrm{Ca}^{2+}$ homeostasis and fiber necrosis. The low extent of necrotic lesions in $N c x 3^{-/-}$skeletal muscles (always $<10 \%$ of total fibers) may result from the quantitatively low intrinsic contribution of NCX3 to the control of $\mathrm{Ca}^{2+}$ efflux in skeletal fibers and/or from the presence of other proteins, which may partially compensate for the absence of NCX3. Among these are NCX1 and PMCA1, two proteins involved in $\mathrm{Ca}^{2+}$ extrusion, which are well expressed in skeletal muscles and, in the case of NCX1, responsible for major cardiac muscle cell necrosis when lacking in mice (8). However, no increased NCX1 or PMCA1 expression was observed in $\mathrm{Ncx}^{-/-}$muscles.

In response to low $\mathrm{Na}^{+}$-containing medium, a rather small reverse exchange was detected in $\mathrm{Ncx}^{+/+}$skeletal fibers. In contrast, no $\mathrm{Ca}^{2+}$ influx was observed in $\mathrm{Ncx}^{-1-}$ fibers in the same condition, suggesting that in these cells, the small reverse exchange is exclusively dependent on NCX3. These unexpected results also suggest that in skeletal muscle fibers, NCX3 is the predominant functional isoform and that NCX1 activity has a strong dependence on $\left[\mathrm{Ca}^{2+}\right]_{i}$, being inactive at low $\left[\mathrm{Ca}^{2+}\right]_{\mathrm{i}}(\sim 50 \mathrm{nM}$; reverse mode $)$ and operative at high $\left[\mathrm{Ca}^{2+}\right]_{\mathrm{i}}(>200 \mathrm{nM}$; forward mode).

For the first time, positive NCX3-ir was detected at the $\mathrm{NMJ}$ and in nerve ramifications between muscle fibers. Although a clear demonstration of the presence of NCX3 protein at the presynaptic level is currently lacking, our results indicate that NCX3 plays an essential role in the control of neuromuscular transmission. Indeed, major EMG abnormalities, including an incremental response and a prominent postexercise facilitation, were recorded at high-frequency nerve stimulation in $\mathrm{Ncx}^{-/-}$muscle. The increment of the muscle fiber response was associated with a facilitation of EPP amplitudes and little depression of synaptic responses. During the 100 -ms period after the high-frequency nerve stimulation, there was an elevated quantal delayed release in $\mathrm{Nc} \times 3^{-/-}$NMJs that was five to six times higher than in $\mathrm{Ncx}^{+/+} \mathrm{NMJs}$. SFEMG also revealed abnormal jitter, frequent neuromuscular blocks, and MCD improvement at higher discharge rate in the mutant mice.

Combined with NCX3 localization at the NMJ and the facilitation of the EMG and contractile responses at HRRS, our electrophysiological results suggest that $\mathrm{Ca}^{2+}$ entering motor nerve terminals may be cleared relatively slower in the absence of the NCX3, thereby enhancing residual $\mathrm{Ca}^{2+}$ and evoked and delayed quan- 
tal transmitter release during RNS. Recent results from the analysis of mice lacking NCX2 support this hypothesis. Indeed, Jeon et al. have recently observed a delayed $\mathrm{Ca}^{2+}$ clearance from $\mathrm{Ncx} 2^{-/-}$hippocampal pyramidal neurons following glutamate stimulation (9). The slower recovery of $\left[\mathrm{Ca}^{2+}\right]_{i}$ to basal level in presynaptic terminals of their mutant mice is associated with an enhanced short- and long-term potentiation (9).

According to Blaustein et al., $\mathrm{Na} / \mathrm{Ca}$ exchange could reverse during membrane depolarization and contribute to $\mathrm{Ca}^{2+}$ entry in nerve cells $(34,35)$. We propose that in mice lacking $\mathrm{NCX} 3$, a decreased $\mathrm{Ca}^{2+}$ influx through the latter pathway may explain the low amplitude CMAPs and the decremental electromyographic responses observed at LRRS.

The main clinical features associated with impaired neuromuscular transmission are weakness and fatigability. Using wire test and Rota-Rod assays, we demonstrated that $\mathrm{Ncx}^{---}$mice have reduced activity, weakness of forelimb muscles, and readily fatigue in comparison to $\mathrm{Ncx}^{+/+}$mice. However, since NCX3 is also expressed in the central and peripheral nervous system, these symptoms cannot exclusively be attributed to alterations at the NMJ or the skeletal fiber and may also partially or totally result from defects in the nervous system.

In this study, we attempted to define the role of NCX3 in neuromuscular transmission and in skeletal muscles in vitro and in vivo using mice lacking NCX3. Our data indicate that $\mathrm{Ncx}^{-/-}$mice present an impaired neuromuscular transmission and a slight skeletal muscle fiber necrosis, highlighting the important role of this exchanger in motor nerve terminals and muscle fibers.

\section{Acknowledgments}

We thank K. Gillard, Huy Nguyen Tran, O. Giot, E. Marion, and S. Mellaerts for technical contributions and J.D. Franssen, J.M. Raymackers, A. de Kerchove, A. Kamagate, and Y. Sokolow for discussion. S. Sokolow was supported by the Belgian National Fund for Scientific Research (FNRS), the Belgian Telethon, and the Fondation Rose et Jean Hoguet and is a research fellow at the FNRS. S. Schurmans and J.-M. Vanderwinden are Senior Research Associates of the FNRS, and M. Manto is supported by the FNRS. This work was supported by the Belgian Telethon, a grant from the Université Libre de Bruxelles, by the Fonds de la Recherche Scientifique Médicale de Belgique (to S. Schurmans), by the Association française contre les myopathies (AFM) (to P. Gailly and J. Molgó), by an Action de Recherche Concertée grant (00/05-260) from the General Direction of Scientific Research of the French Community of Belgium (to P. Gailly) and by the Fondation Médicale Reine Elisabeth and the Fondation Universitaire David and Alice Van Buuren (to J.-M. Vanderwinden).

1. Clapham, D.E. 1995. Calcium signaling. Cell. 80:259-268.

2. Blaustein, M.P., and Lederer, W.J. 1999. Sodium/calcium exchange: its physiological implications. Physiol. Rev. 79:763-854.

3. Philipson, K.D., and Nicoll, D.A. 1992. Sodium-calcium exchange. Curr. Opin. Cell Biol. 4:678-683.

4. Nicoll, D.A., Longoni, S., and Philipson, K.D. 1990. Molecular cloning and functional expression of the cardiac sarcolemmal $\mathrm{Na}(+)-\mathrm{Ca} 2+$ exchanger. Science. 250:562-565.

5. Nicoll, D.A., et al. 1996. Cloning of a third mammalian $\mathrm{Na}+-\mathrm{Ca} 2+$ exchanger, NCX3. J. Biol. Chem. 271:24914-24921.

6. Li, Z., et al. 1994. Cloning of the NCX2 isoform of the plasma membrane $\mathrm{Na}(+)-\mathrm{Ca} 2+$ exchanger. J. Biol. Chem. 269:17434-17439.

7. Quednau, B.D., Nicoll, D.A., and Philipson, K.D. 1997. Tissue specificity and alternative splicing of the $\mathrm{Na}+\mathrm{Ca} 2+$ exchanger isoforms NCX1, NCX2, and NCX3 in rat. Am. J. Physiol. 272:C1250-C1261.

8. Wakimoto, K., et al. 2000. Targeted disruption of $\mathrm{Na}+\mathrm{Ca} 2+$ exchanger gene leads to cardiomyocyte apoptosis and defects in heartbeat. J. Biol. Chem. 275:36991-36998.

9. Jeon, D., et al. 2003. Enhanced learning and memory in mice lacking $\mathrm{Na}+/ \mathrm{Ca} 2+$ exchanger 2. Neuron. 38:965-976.

10. Fraysse, B., et al. 2001. Expression of the $\mathrm{Na}(+) / \mathrm{Ca}(2+)$ exchanger in skeletal muscle. Am. J. Physiol. Cell Physiol. 280:C146-C154.

11. Deval, E., Levitsky, D.O., Constantin, B., Raymond, G., and Cognard, C. 2000. Expression of the sodium/calcium exchanger in mammalian skeletal muscle cells in primary culture. Exp. Cell Res. 255:291-302.

12. De Backer, F., Vandebrouck, C., Gailly, P., and Gillis, J.M. 2002. Long-term study of $\mathrm{Ca}(2+)$ homeostasis and of survival in collagenase-isolated muscle fibres from normal and $\mathrm{mdx}$ mice. J. Physiol. 542:855-865.

13. Balnave, C.D., and Allen, D.G. 1998. Evidence for $\mathrm{Na}+/ \mathrm{Ca} 2+$ exchange in intact single skeletal muscle fibers from the mouse. Am. J. Physiol. 274:C940-C946.

14. Vanderwinden, J.M., Rumessen, J.J., Bernex, F., Schiffmann, S.N., and Panthier, J.J. 2000. Distribution and ultrastructure of interstitial cells of Cajal in the mouse colon, using antibodies to Kit and Kit(W-lacZ) mice. Cell Tissue Res. 302:155-170.

15. Vanderwinden, J.M., Rumessen, J.J., De Laet, M.H., Vanderhaeghen, J.J., and Schiffmann, S.N. 2000. CD34 immunoreactivity and interstitial cells of Cajal in the human and mouse gastrointestinal tract. Cell Tissue Res. 302:145-153.

16. Vilquin, J.T., et al. 1998. Evidence of mdx mouse skeletal muscle fragility in vivo by eccentric running exercise. Muscle Nerve. 21:567-576.

17. Lin, T.S., and Cheng, T.J. 1998. Stimulated single-fiber electromyography in the rat. Muscle Nerve. 21:482-489.

18. Gomez, C.M., et al. 1997. Slow-channel transgenic mice: a model of postsynaptic organellar degeneration at the neuromuscular junction. J. Neurosci. 17:4170-4179.

19. Verschuuren, J.J., Spaans, F., and De Baets, M.H. 1990. Single-fiber electromyography in experimental autoimmune myasthenia gravis. Muscle Nerve. 13:485-492.

20. Gooch, C.L., and Mosier, D.R. 2001. Stimulated single fiber electromyography in the mouse: techniques and normative data. Muscle Nerve. 24:941-945.

21. Oh, J. 1998. Principles of clinical electromyography case studies. Lippincott Williams \& Wilkins. Baltimore, Maryland, USA. 604 pp.

22. Trontelj, J.V., and Stalberg, E. 1991. Single motor end-plates in myasthenia gravis and LEMS at different firing rates. Muscle Nerve. 14:226-232.

23. Sanders, D.B. 1992. The effect of firing rate on neuromuscular jitter in Lambert-Eaton myasthenic syndrome. Muscle Nerve. 15:256-258.

24. Minic, J., Chatonnet, A., Krejci, E., and Molgó,J. 2003. Butyrylcholinesterase and acetylcholinesterase activity and quantal transmitter release at normal and acetylcholinesterase knockout mouse neuromuscular junctions. Br. J. Pharmacol. 138:177-187.

25. McLachlan, E.M., and Martin, A.R. 1981. Non-linear summation of endplate potentials in the frog and mouse. J. Physiol. 311:307-324.

26. Cruz, L.J., et al. 1985. Conus geographus toxins that discriminate between neuronal and muscle sodium channels. J. Biol. Chem. 260:9280-9288.

27. Ten Hage, L., et al. 2002. Effects of toxic extracts and purified borbotoxins from Prorocentrum borbonicum (Dinophyceae) on vertebrate neuromuscular junctions. Toxicon. 40:137-148.

28. Raymackers, J.M., et al. 2003. Consequence of parvalbumin deficiency in the mdx mouse: histological, biochemical and mechanical phenotype of a new double mutant. Neuromuscul. Disord. 13:376-387.

29. Zacharias, D.A., and Kappen, C. 1999. Developmental expression of the four plasma membrane calcium ATPase (Pmca) genes in the mouse. Biochim. Biophys. Acta. 1428:397-405.

30. Donoso, P., and Hidalgo, C. 1989. Sodium-calcium exchange in transverse tubules isolated from frog skeletal muscle. Biochim. Biophys. Acta. 978:8-16.

31. Hidalgo, C., Gonzalez, M.E., and Garcia, A.M. 1986. Calcium transport in transverse tubules isolated from rabbit skeletal muscle. Biochim. Biophys. Acta. 854:279-286.

32. Westerblad, H., and Allen, D.G. 1991. Changes of myoplasmic calcium concentration during fatigue in single mouse muscle fibers. J. Gen. Physiol. 98:615-635.

33. Lynch, G.S., Fary, C.J., and Williams, D.A. 1997. Quantitative measurement of resting skeletal muscle [Ca2+]i following acute and long-term downhill running exercise in mice. Cell Calcium. 22:373-383.

34. Blaustein, M.P., et al. 1991. Physiological roles of the sodium-calcium exchanger in nerve and muscle. Ann. N. Y. Acad. Sci. 639:254-274.

35. Luther, P.W., et al. 1992. Presynaptic localization of sodium/calcium exchangers in neuromuscular preparations. J. Neurosci. 12:4898-4904. 